\title{
Un nouveau mode de régulation pour la glucokinase hépatique
}

De faibles concentrations de fructose stimulent la phosphorylation du glucose dans le foie. Cet effet met en jeu une protéine régulatrice qui, en présence de fructose-6-phosphate, inhibe la glucokinase en formant un complexe avec cette enzyme. En se liant à la protéine régulatrice, le fructose-1-phosphate, un métabolite spécifique du fructose, provoque la dissociation du complexe, permettant à la glucokinase d'exprimer pleinement son activité. $L$ 'existence de ce mécanisme amène à postuler que le fructose joue un rôle de signal dans le contrôle de l'utilisation du glucose par le foie.

\section{Émile Van Schaftingen Annick Vandercammen Michel Detheux}

\section{ADRESSE}

E. Van Schaftingen : maitre de recherches $d u$ FNRS. A. Vandercammen : aspirant UCL. M. Detheux : boursier IRSIA. Laboratoirc de chimic physiologique, universitć catholique de Louvain et International institute of cellular and molecular pathology, avenuc Hip-

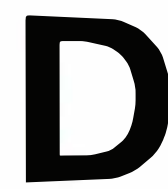

ans le monde animal, la conversion du glucose en glucose-6-phosphate cst catalysée par quatre hexokinases différentes. Présentes à des degrés divers dans la plupart des tissus, les hexokinases I, II et III sont très semblables ct sc caractérisent par leur très hautc affinité pour le glucose $(\mathrm{Km} \leqslant 0,2 \mathrm{mM})$ ct par le fait qu'elles sont inhibées par des concentrations physiologiques de glucose-6-phosphate [1]. Lcur activité est donc automatiquement contrôléc par celles des voics métaboliques qui utilisent ou forment du glucose-6-phosphate : glycolyse, cycle des pentoses-phosphate, synthèse et dégradation du glycogène.

La quatrième hexokinase, la glucokinase [2], se distingue par une affinité beaucoup plus faible pour le glucose, lc $\mathrm{Km}$ se situant aux alentours de $10 \mathrm{mM}$, une valeur légèrement supérieure au niveau normal de la glycémie. De plus, la courbe de saturation pour le glucose est sigmoïde, ce qui cst inhabituel pour une enzyme exis- tant à l'état de monomère. Cettc particularité cinétique scmble due au fait que l'enzyme posséderait plus d'affinité pour le glucose immédiatement après un cycle catalytique, que lorsqu'elle n'a plus " vu " le glucose depuis longtemps [3]. En quelque sorte, la glucokinase "se souvient" pendant un certain temps (de l'ordre de la milliseconde) d'avoir converti unc molécule de glucose en glucose-6-phosphatc. Ce genre de mécanisme est connu sous la dénomination de modèle mnémonique.

Unc deuxic̀me différence importante entre la glucokinase et les autres hexokinases réside dans le fait que la glucokinase n'est pas inhibée par des concentrations physiologiques de glucose-6-phosphatc. De plus, sa taille est égale à pratiquement la moitié de celle des autres hexokinases (55 contre $100 \mathrm{kDa}$ ). Les gènes codant pour ces dernières semblent résulter de la duplication d'un gène ancestral, suivic d'une fusion et de l'évolution d'une des deux parties en un domaine régulateur comportant un 
site allostérique pour le glucose-6-phosphate [4].

Enfin, la glucokinase peut être inhibée, du moins à l'ćtat d'enzyme purifiée, par les acyl-CoA à longue chaîne [5]. Toutefois, il n'existe, à notre connaissance, aucune indication de ce que cette inhibition s'exerce physiologiquement.

\section{Rôle de la glucokinase dans le foie et les cellules $\beta$}

Son affinité relativement faible pour le glucose et sa courbe de saturation sigmoïde font que la glucokinase est à même de répondre à des changements de la glycémie par des variations de son activité. On ne s'étonnera donc pas que cette hexokinase particulière se retrouve principalement dans deux types de cellules qui jouent un rôle important dans le contrôle de la glycémie : les cellules parenchymateuses du foie et les cellules $\beta$ des îlots de Langerhans [2, 6]. Ces deux types de cellules sont dotées d'un transporteur pour le glucose (Glut 2, [7]) tellement actif que la concentration intracellulaire de celuici y est à tout instant quasi égale à celle du plasma.

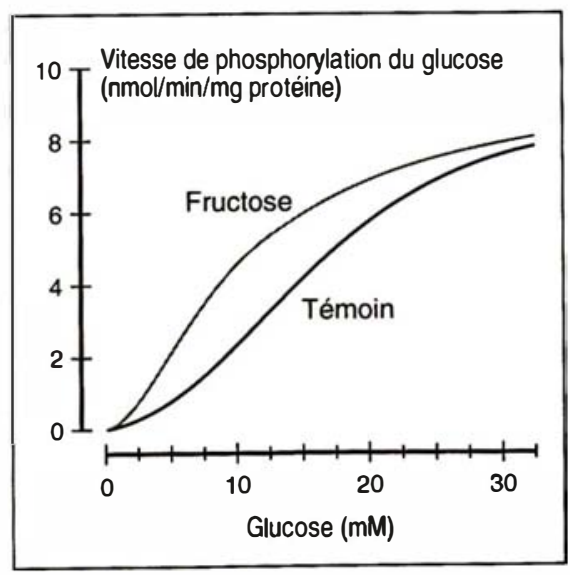

Figure 1. Effet de l'addition de fructose sur la vitesse de phosphorylation du glucose par des hépatocytes isolés en suspension. La vitesse de phosphorylation du glucose a été déterminée par la libération d'eau tritiée à partir de glucose $[2-3 \mathrm{H}]$. Le fructose a été ajouté à une concentration de 0,2 mM. (D'après [14].)

$\mathrm{m} / \mathrm{s} n^{\circ} 1$, vol. 8 , janvier 92
Dans le contrôle de la glycémie, le foie joue un rôle d'organe effecteur : capable d'utiliser le glucose en pćriode post-prandiale, entrc autres pour le stocker sous forme de glycogène, il en produit au cours du jeûne. Ce processus est contrôlé par diverses hormones (glucagon, insuline, catécholamines...) ainsi que par la concentration plasmatique de glucose. Celle-ci, d'une part, module la vitesse de phosphorylation du glucose par la glucokinase et, d'autre part, influence la synthèse de glycogène : la fixation du glucose sur la glycogène phosphorylase (responsable de la dégradation du glycogène) entraîne l'inactivation de cette enzyme ainsi que l'activation de la glycogène synthase $[8,9]$.

Selon les vues actuelles, c'est la vitesse du mćtabolisme du glucose qui, dans la cellule $\beta$ de l'îlot de Langerhans, contrôle la sécrétion insulinique. Cette vitesse dépendant de l'activité de la glucokinase, il semble bien que cette dernière soit le glucose sensor longtemps recherché $[6,10]$. Nous n'évoquerons pas dans le détail les recherches actuelles sur la structure primaire et la régulation de l'expression de la glucokinase. Rappelons simplement qu'il existe de petites différences dans la séquence en acides aminés, principalement au niveau de l'extrémité $\mathrm{N}$-terminale, entre la glucokinase des cellules $\beta$ du pancréas et l'enzyme hépatique. Par ailleurs, l'expression de la glucokinase est, dans le foie, stimulée par l'insuline et inhibée par le glucagon. Dans la cellule $\beta$, elle serait sous le contrôle de la concentration de glucose [11].

\section{Régulation à court terme de l'activité de la glucokinase dans les hépatocytes}

Jusqu'il y a peu, il semblait donc que l'activité de la glucokinase n'était contrôlée, à court terme, que par la concentration de glucose. Cette vue simpliste des choses ne permettait cependant pas de rendre compte de deux observations réalisées sur des suspensions d'hépatocytes isolés, modèle communément utilisé pour l'étude des fonctions métaboliques de la cellule hépatique. En 1978, Bontemps et al. [12] ont mesuré l'activité de la glucokinase dans des hépatocytes par la libération d'eau tritiée à partir de glucose $\left[2-{ }^{3} \mathrm{H}\right]$; ils ont montré que l'affinité de cette enzyme pour le glucose ćtait notablement plus faible que celle de l'enzyme purificee, mais qu'elle augmentait de près de deux fois si l'on incubait les cellules dans un milieu riche en potassium $(144 \mathrm{mM})$ et pauvre en sodium ( $5 \mathrm{mM}$ ) au lieu d'utiliser un milieu Krebs-Ringer. Tout se passait comme si les cellules contenaient un inhibiteur compétitif, qui aurait été neutralisé en milieu riche en potassium. L'année suivante, des chercheurs australiens [13] trouvaient que l'addition de fructose, à concentration modérée (2,5 mM), augmentait, elle aussi, la vitesse de détritiation du glucose $\left[2-{ }^{3} \mathrm{H}\right]$.

Partant de cette dernière observation, nous avons trouvé que l'effet du fructose s'observait dé jà à des concentrations très faibles $(<0,1 \mathrm{mM})$, ce qui plaidait en faveur de sa signification physiologique. Par ailleurs, comme dans le cas de l'effet "potassium " mentionnć plus haut, l'addition de fructose semblait augmenter l'affinité de la glucokinase pour son substrat à l'intérieur même des cellules (figure 1). Enfin, le sorbitol et le Dglycéraldéhyde avaient un effet stimulateur analogue à celui du fructose, alors que la dihydroxyacétone en était dépourvue. Cela indiquait que l'effet stimulateur était exercé par un métabolite formć à partir de fructose, de sorbitol ou de D-glycéraldéhyde, mais non à partir de dihydroxyacétone [14]. Comme on peut le déduire du schéma métabolique présenté dans la figure 2, le métabolite qui semblait être le meilleur candidat était le fructose-1-phosphate, un intermédiaire normal du métabolisme du fructose et du sorbitol, qui peut être aussi formé à partir de Dglycéraldéhyde, par condensation avec la dihydroxyacétone-phosphate. Restait à montrer que le fructose-1-phosphate agissait sur la glucokinase.

\section{Découverte de la protéine régulatrice}

Toutes les expériences visant à montrer un effet stimulateur du fructose-1-phosphate sur de la glucokinase 
purifiée sont restées sans succès. En revanche, le fructose-1-phosphate stimulait la phosphorylation du glucose dans des extraits de foie bruts. Mises ensemble, ces deux observations indiquaient que la régulation de la glucokinase par le fructose-1-phosphate impliquait un facteur qui était perdu au cours de la purification de l'enzyme. Nous avons pu confirmer cette hypothèse en montrant que la chromatographie d'un extrait de foie sur échangeur d'anions séparait la glucokinase d'une protéine qui inhibait cette enzyme en absence de fructose-1-phosphate mais non en sa présence [15]. Après purification de cette protéine, nous nous sommes aperçus de ce que le fructose-6-phosphate accentuait considérablement son effet inhibiteur alors que le glucose-6-phosphate restait sans effet. La protéine régulatrice permet donc le contrôle de la glucokinase par deux effecteurs ayant une action antago- niste : le fructose-6-phosphate, quı agit comme inhibiteur, et le fructose-1-phosphate, qui a une action activatrice (ou, plus exactement, " désinhibitrice ").

\section{Mode d'action de la protéine régulatrice}

La protéine régulatrice a été purifiée jusqu'à quasi-homogénéité et identifiée à un polypeptide monomérique de $62 \mathrm{kDa}$. Divers arguments expérimentaux indiquent que c'est en formant un complexe avec la glucokinase que la protéine régulatrice l'inhibe. Ainsi, la masse moléculaire apparente de la glucokinase, mesurée en gradient de sucrose, passe d'une valeur de 55 à plus de $100 \mathrm{kDa}$ lorsqu'elle est mise en présence de protéine régulatrice et de fructose-6-phosphate. Cette augmentation de masse apparente ne survient pas en absence de fructose-6-phosphate

4. White TK, Wilson JE. Isolation and characterization of the discrete $\mathrm{N}$ - and $\mathrm{C}$ terminal halves of rat brain hexokinase retention of full catalytic activity in the isolated C-terminal half. Arch Biochem Biophys $1989 ; 274: 375-93$.

5. Dawson CM, Hales CN. The inhibition of rat liver glucokinase by palmitoyl-CoA. Biochim Biophys Acta 1969 ; 176 : 657-9.

6. Lenzen S, Panten U. Signal recognition by pancreatic $\beta$-cells. Biochem Pharmacol $1988 ; 37: 371-8$

7. Gould GW, Bell Gl. Facilitative glucose transportcrs : an expanding family. Trends Biol Sci 1990 ; 15 : 18-23.

8. Hers HG. The control of glycogen metabolism in the liver. Ann Rev Biochem 1976 ; $45: 167-89$.

9. Cohen P. The structure and regulation of protcin phosphatases. Ann Rev Biochem $1989 ; 58: 453-508$

10. Meglasson MD, Matschinsky FM. Pancreatic islet metabolism and regulation of insulin secretion. Diabetes Metab Rev 1986 ; $2: 163-214$.

11. Magnuson MA. Glucokinase gene structure. Functional implications of molccular genetic studics. Diabeles $1990 ; 39$ : 523-7.

12. Bontemps F, Hue L, Hers HG. Phosphorylation of glucose in isolated hepatocytes. Sigmoidal kinctics explained by the activity of glucokinase alone. Biochem $J$ 1978 ; 174 : 603-11.

13. Clark DG, Filsell OH, Topping DL. Effects of fructose concentration on carbohydrate metabolism, heat production inic substrate cycling in isolated rat hepatocytes.

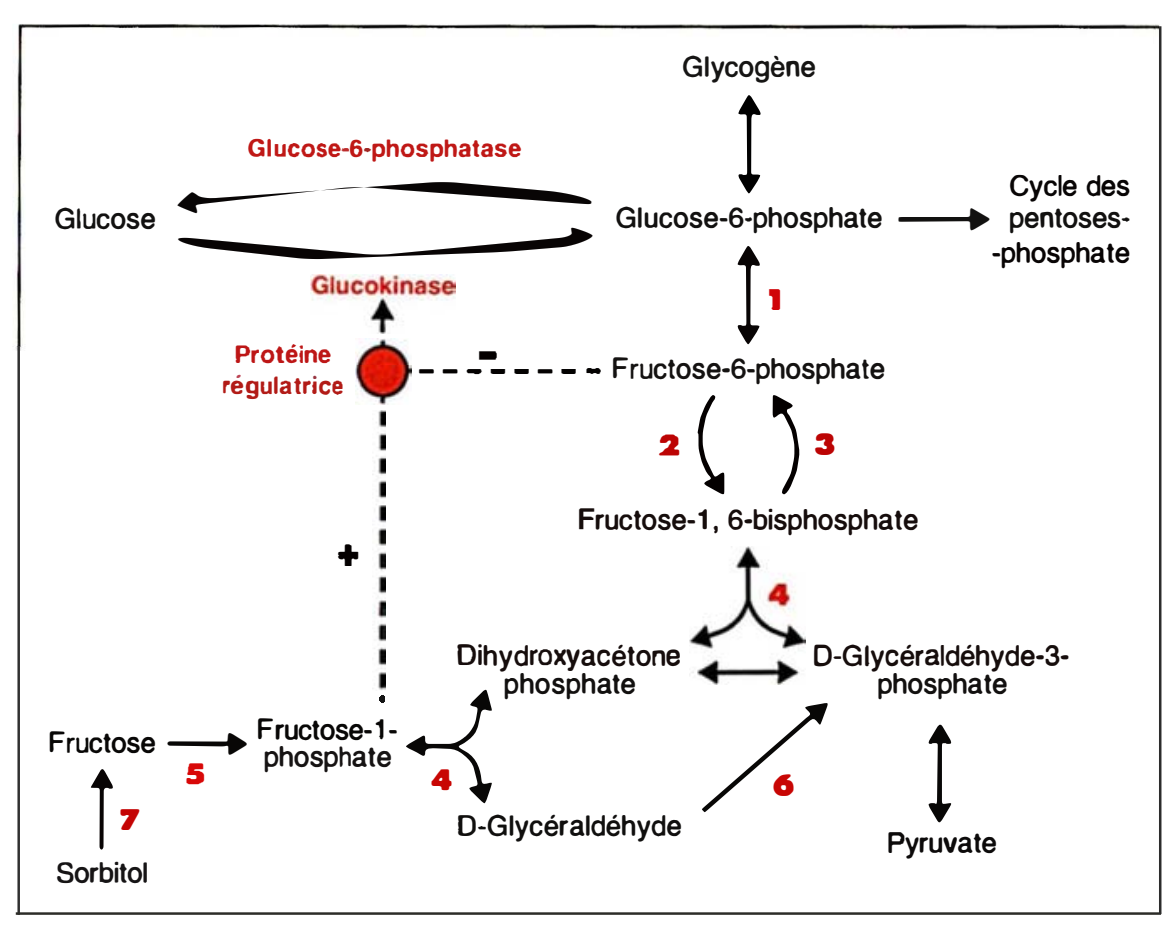

Figure 2. Métabolisme du glucose, du fructose et du D-glycéraldéhyde dans le foie. Les chiffres entre parenthèses indiquent les réactions catalysées par les enzymes suivantes : (1) phosphoglucose-isomérase ; (2) 6-phosphofructo 1-kinase ; (3) fructose 1,6-bisphosphatase ; (4) aldolase ; (5) fructokinase ; (6) triokinase; (7) sorbitol-déshydrogénase. Le schéma ne montre pas que la triokinase convertit aussi la dihydroxyacétone en dihydroxyacétone-phosphate. 


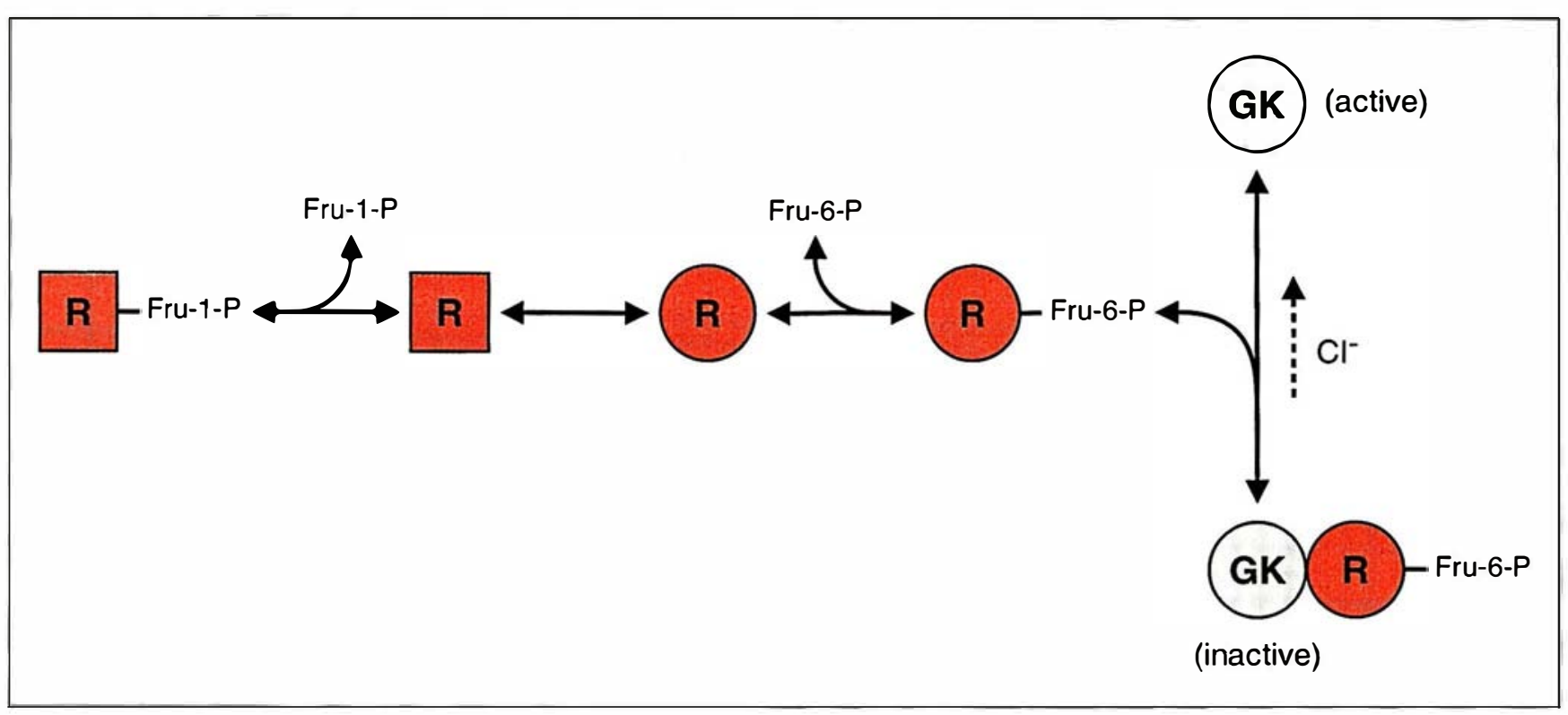

Figure 3. Mécanisme de l'effet du fructose-1-phosphate, du fructose-6-phosphate et des ions CI-. Selon le modèle présenté, la protéine régulatrice $(R)$ existe sous deux conformations différentes. Le fructose-1-phosphate (Fru-1-P) se fixe sur la forme symbolisée par un carré, et le fructose-6-phosphate (Fru-6-P), sur celle représentée par un cercle. $C^{\prime}$ est seulement sous cette dernière conformation que la protéine régulatrice peut former un complexe inactif avec la glucokinase. Les ions $\mathrm{Cl}^{-}$agissent en empêchant la formation de ce complexe.

ou lorsque du fructose-1-phosphate est présent en plus du fructose-6-phosphate. On observe aussi la réciproque, à savoir que la présence de glucokinase et de fructose-6-phosphate élève la masse moléculaire apparente de la protéine régulatrice [16].

Le fructose-6-phosphate et le fructose-1-phosphate modulent donc l'activité de la glucokinase, le premier en favorisant et le second en prévenant la formation du complexe que cette enzyme forme avec la protéine régulatrice. Les deux esters phosphoriques agissent en se liant à celle-ci et, selon toute vraisemblance, en induisant des changements conformationnels. La figure 3 nous montre le modèle qui rend le mieux compte des observations faites jusqu'à présent. Mentionnons que d'autres composés que le fructose-1-phosphate lèvent, à concentration plus élevée que ce dernier, l'inhibition exercée par la protéine régulatrice. Certains d'entre eux agissent de façon compétitive vis-à-vis du fructose-6-phosphate (phosphate inorganique, divers esters phosphoriques [17]), et d'autres, de manière non compétitive (chlorure et autres $\mathrm{m} / \mathrm{s} n^{\circ} 1$, vol. 8 , janvier 92 anions monovalents [18]).

L'autre aspect de la question est de comprendre comment la protéine régulatrice, en se fixant à la glucokinase, exerce une inhibition compétitive de cette dernière vis-à-vis du glucose. En règle générale, les composés qui possèdent une certaine analogie de structure avec le substrat et se fixent à sa place au niveau du site catalytique exercent une inhibition de type compétitif (c'est-à-dire que l'on peut lever en augmentant la concentration de substrat). Il existe aussi des inhibiteurs compétitifs qui agissent en se fixant à distance du site catalytique. Dans le cas de la glucokinase, la glucosamine et la $\mathrm{N}$-acétylglucosamine sont à ranger parmi les analogues de substrat, et les acyl-CoA à longue chaîne, parmi les effecteurs allostériques. Une étude cinétique de l'effet de combinaisons d'inhibiteurs sur l'activité de la glucokinase a indiqué que la liaison de la protéine régulatrice à la glucokinase ne se ferait pas sur le site catalytique mais vraisemblablement sur le site de fixation des acyl-CoA [18]. Cette conclusion est en accord avec le fait que l'inhibition par la protéine régulatrice et celle exercée par les acyl-CoA à longue chaîne se retrouvent conjointement dans toutes les glucokinases animales que nous avons testées, mais qu'aucune des deux ne s'observe avec les hexokinases d'origine animale ou de microorganismes [18]. Cette parenté entre les effets des acyl-CoA et de la protéine régulatrice suggère que cette dernière interagirait avec la glucokinase via un groupement hydrophobe (par exemple, un groupement myristyle).

\section{Avantages de la régulation par le fructose-1-phosphate}

Le modèle décrit plus haut permet de rendre compte de la régulation de la glucokinase in vivo. En période postabsorptive, la concentration de fructose-1-phosphate est extrêmement faible dans le foie, en dessous du seuil de détection de nos méthodes de mesure (quelques $\mathrm{nmol} / \mathrm{g}$ de tissus $[19,20])$. Le fructose-6-phosphate étant présent, la protéine régulatrice peut exercer son action inhibitrice. Cela explique que l'affinité apparente de la glucokinase pour le glucose soit 


\section{RÉFÉRENCES}

14. Van Schaftingen E, Vandercammen A Stimulation of glucosc phosphorylation by fructose in isolated hepatocytes. Eur J Bio chem $1989 ; 179: 173-7$.

15. Van Schaftingen E. A protein from rat liver confers to glucokinase the property of bcing antagonistically regulated by fructosc 6-phosphatc and fructose 1-phosphatc. Eur $J$ Biochem 1989 ; 179 : 179-84

16. Vandercammen A, Van Schaftingen E. The mechanism by which rat liver glucokinase is inhibited by the regulatory protein. Eur J Biochem 1990 ; 191 : 483-9.

17. Detheux M, Vandercammen A, Van Schaftingen E. Effectors of the regulatory protein acting on liver glucokinase : a kinctic investigation. Eur J Biochem 1991; 200 553-61.

18. Vandercammen A, Van Schaftingen E. Competitive inhibition of liver glucokinase by its regulatory protcin. Eur $J$ Biochem $1991 ; 200$ : 545-51.

19. Davics DR, Detheux M, Van Schaftingen E. Fructose 1-phosphate and the regulation of glucokinase activity in isolated hepatocytes. Eur J Biochem 1990; 192 : 283-9.

20. Van Schaftingen E, Davies DR. Fructose administration stimulates glucose phosphorylation in the livers of anacsthetized rats. FASEB J 1991; 5 : 326-30.

21. Hers HG. Le métabolisme du fructose Bruxclles : Arscia, 1958 : 56.

22. Scglen PO. Autorcgulation of glycolysis, respiration, gluconcogenesis and glycogen synthesis in isolated parenchymal rat liver cells under acrobic and anacrobic conditions. Biochim Biophys Acta 1974; 338 : 317-36.

23. Topping DL, Maycs PA. The immediate effects of insulin and fructose on the metabolism of the perfused liver. Changes in lipoprotcin secretion, fatty acid oxidation and csterification, lipogenesis and carbohydratc metabolism. Biochem J $1972 ; 126$ : 295-311.

24. Topping DL, Maycs PA. The concentrations of fructose, glucose and lactate in the splanchnic blood vessels of rats absorbing fructosc. Nutr Metab $1971 ; 13$ : 331-8.

25. Corvilain J, Tagnon R. Effects of fruc tose infusion on glucose uptake and circulating insulin-like activity in normal men. plus faible dans le cas de l'enzyme présentc dans des hépatocytes isolés que dans le cas de l'enzyme purifiée $[12,14]$, et que chez lc rat anesthésié, la vitesse de phosphorylation du glucose par le foic ne représente qu'à peu près $40 \%$ de l'activité potentielle de la glucokinasc, mesuréc dans des extraits [20]. Lc fructose, en provoquant la formation de fructose-1-phosphate, supprime l'inhibition exercée par la protéine régulatrice : l'affinité de la glucokinase dans les hépatocytes devient comparablc à celle de l'enzyme purifiée [14] ; chez le rat anesthésié, la vitesse de phosphorylation du glucose augmente de près de trois fois sous l'action du fructosc, atteignant la valeur attendue à partir des mesures faites dans un extrait [20].

Les doses de fructose requises pour obsẹrver cet effet stimulateur chez le rat anesthésié sont relativement faibles, un doublement de la vitesse de phosphorylation s'observant déjà avec unc dose per os de $20 \mathrm{mg} / \mathrm{kg}$ (figure 4), soit, extrapolć à l'homme, l'ćquivalent d'un gramme et demi pour un adulte de $70 \mathrm{~kg}$. On peut donc conclure qu'un apport modéré de fructose stimule l'utilisation du glucose

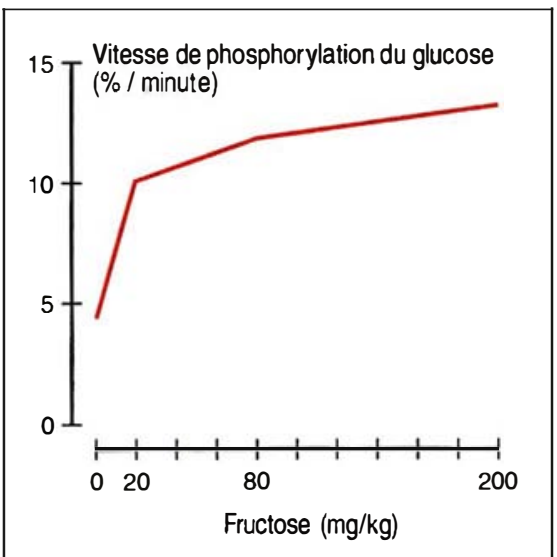

Figure 4. Effet du fructose sur la vitesse de phosphorylation du glucose dans le foie de rat. Les animaux ont reçu par gavage la dose de fructose indiquée et ont ensuite été anesthésiés. La vitesse de libération $d^{\prime}$ eau tritiée à partir de glucose [2-3H] a été déterminée 20 minutes après I'administration de fructose. (D'après [20].) par le foic. Cettc conclusion est en accord avec des observations ancienncs montrant que le fructose accélère la conversion du glucose en glycogène hépatique $[21,22]$. Elle permet aussi de rendre compte du léger effet hypoglycémiant du fructose observé chez l'homme et chez le rat [23-25]. (Cette hypoglycémie modérée n'a rien à voir avec l'hypoglycémie profonde obscrvéc chez les individus souffrant d'intolérance au fructose. Dans cc cas, l'hypoglycémie est due au blocage de la néoglucogenèse et de la dégradation de glycogène suite à l'accumulation de concentrations plusicurs fois millimolaires de fructose-1-phosphate [26].)

La nature a donc pourvu la cellule hépatique d'un mécanisme spécifique permettant au fructose de faciliter l'utilisation du glucosc. Quelle peut être la signification physiologique de cette "collaboration " entre glucose et fructose ? Nous pensons que le fructose est utilisé comme un signal qui permet au foie de mieux utiliser le glucose d'origine alimentaire. Le sang portc contient, chez lc sujet à jeun, unc concentration appréciable de glucose $(\approx 5 \mathrm{mM})$. Du fait de ce niveau de départ assez élevé, l'augmentation relative de la glycémie lors de l'absorption d'un repas est faible. Si la concentration de glucose passc, par exemple, de 5 à $7 \mathrm{mM}$, on s'attend que l'activité de la glucokinase n'augmente que d'à peu près $40 \%$, ce qui, en termes de contrôle métabolique, représente peu de chosc.

La plupart des aliments d'origine végétale conticnnent, en plus du glucose, une quantité appréciablc de fructose qui est, soit sous forme libre, soit combiné avec le glucose dans la molécule de saccharosc (les farines constituent une exception importante à cette règle, mais on pcut considérer qu'clles ne sont devenues un aliment important que pour l'homme, ct ce, relativement récemment au cours de l'évolution de l'humanité). Le fructose étant absent du sang porte en période de jeûne, son apparition suite à un repas va provoquer une augmentation de 2 à 3 fois de la vitesse de phosphorylation du glucose. Sa présence dans le sang porte est, en quelque sorte, la signature de ce que l'intestin absorbe des hydrates de carbone, principalement du glucose. 


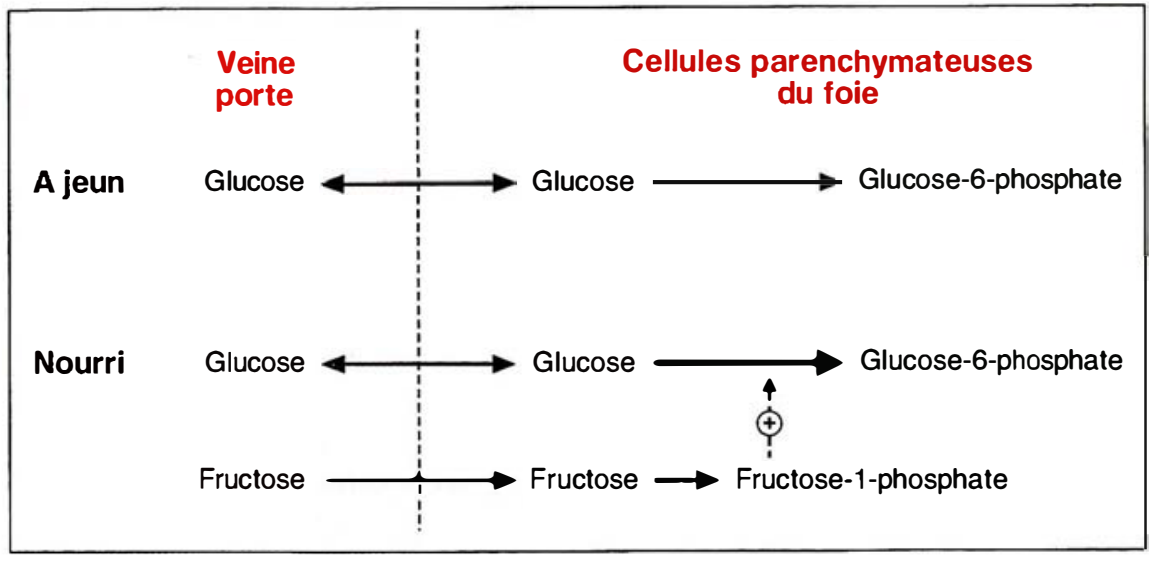

Figure 5. Rôle de l'effet du fructose sur la phosphorylation du glucose. Chez l'animal à jeun, le sang porte ne contient pas de fructose, le glucose étant donc phosphorylé lentement. Le sang porte de l'animal nourri contient du fructose qui, par le truchement de la protéine régulatrice et du fructose-1-phosphate, accélère la phosphorylation du glucose.

Il existe actuellement une controverse (glucose paradox [27]) dans la littérature quant au chemin suivi par lc glucose alimentaire pour devenir du glycogène hépatique. Classiquement, on admet que le glucose est phosphorylé par la glucokinase et dircctement converti en glycogène. Il semble cependant qu'une fraction non négligeable du glycogène provienne de molécules de glucose qui ont été préalablement dégradées en composés à trois carbones (lactate, pyruvate) dans les tissus périphériques et reconvertics en glucose-6-phosphatc par la néoglucogenèse $[27,28]$. Ce grand détour n'est pas surprenant dans la mesure où la plupart des expériences qui le démontrent ont été réalisécs : (1) avec des animaux à jeun, chez lesquels l'activité de la glucokinase est diminuée et (2) à l'aide d'une chargc de glucose pur. Au vu de ce qui est discuté plus haut, il serait intéressant de refaire ces expériences en utilisant un mélange de glucose et de fructose. Dans le même ordre d'idées, on peut se demander dans quelle mesure une consommation modérée de fructose pourrait améliorer la tolérance glucidique des diabétiques.

Il est connu qu'une alimentation riche en fructose ou en sucrose augmente la synthèse hépatique de triglycérides ct la concentration de ces derniers dans le plasma $[29,30]$. Cet cffet résulte d'une augmentation de $\mathrm{m} / \mathrm{s} n^{\circ} 1$, vol. 8 , janvier 92 impliquées dans la lipogenèsc hépatique. Il est vraisemblable que cct effet du fructose, fasse lui aussi, intervenir une accélération de la phosphorylation du glucose.

\section{Mécanisme de l'effet du potassium}

Nous avons mentionné plus haut que le fait d'incuber des hépatocytes isolés dans un milieu riche en potassium stimulait aussi la phosphorylation du glucose. Dans ce cas, la levée de l'effet inhibiteur exercé par la protéine régulatrice n'est pas due à la formation de fructose-1-phosphate, mais cst sccondaire au gonflement ccllulaire ou, plus précisément, à la dilution de la protéine régulatrice et à l'augmentation de la concentration intracellulairc de chlorurc qu'il entraîne (résultats non publiés). Nous avons vu plus haut que l'ion chlorure a la propriété de contrecarrer l'action de la protéine régulatrice. L'effet d'un milieu riche en potassium n'a, bien sûr, aucune signification physiologique voire pathologique. Il souligne cependant l'importance potentielle de modifications ioniques pour le contrôle de l'activité de la glucokinase. Historiquement, il a eu aussi le mérite d'attirer l'attention sur la régulation de cette enzymc. l'activité de différentes enzymes

\section{La protéine régulatrice dans les îlots de Langerhans}

omme nous l'avons mentionné plus haut, la glucokinase est présente dans les cellules $\beta$ des îlots de Langerhans, où ellc scmblc joucr un rôlc important dans le contrôle du flux glycolytique et, par cc biais, dans la sécrétion insulinique. Divers arguments indirects indiquent que la protéine régulatrice est présente dans les îlots [31]. Tout d'abord la glucokinasc d'îlots est, malgré ses petites différences de structure d'avec l'enzyme de foic (voir plus haut), sensiblc à la protéinc régulatrice d'origine hépatique. En dcuxième lieu, la phosphorylation du glucose dans des homogénats d'îlots est (modestement) stimulée par le fructose-1-phosphate. Enfin, lc D-glycéraldéhyde, qui augmente la concentration de fructosc-1phosphate dans les îlots, stimulc la phosphorylation du glucose. Il est à remarquer que le fructose n'a pas d'cffet significatif, ce qui n'est pas surprenant dans la mesure où l'activité de la fructokinase est tellement faible dans les îlots qu'il n'y a guère de fructose-1-phosphate qui se forme à partir du cétosc. Dans l'état actuel de nos connaissances, on comprend donc mal le rôlc que jouc la protéinc régulatrice dans lcs îlots. Il n'en reste pas moins que les cellules $\beta$ sont dotées d'un mécanisme permettant, en théorie au moins, de moduler leur sensibilité au glucose.

\section{Conclusion}

Une des leçons que nous apprend la découverte de cette protéine régulatrice cst que la régulation métabolique est plus compliquéc que nous le pensions. Dans une large mesure, les propriétés cinétiques des enzymes ont été étudiées sur des préparations purifiées ou dans des extraits tissulaires fortement dilués. Dans ces conditions, on met en évidence sans trop de peine les propriétés régulatrices intrinsèques d'une protéine, mais on court le risque de passer à côté de celles qui lui sont conférées par une protéine distincte, soit parce que celle-ci est perduc au cours de la purification, soit parce qu'elle se lie avec trop peu d'affinité pour exercer ses effets dans un système dilué. 


\section{RÉFÉRENCES}

26. Gitzclmann R, Stcinmann B, Van den Berghe G. Disorders of carbohydrate metabolism. In : Scriver CR, Bcaudet AL, Sly WS, Valle D, cds. The Metabolic Basis of Inherited Disease. 6e éd. New York : McGraw Hill, 1989 : 399-424.

27. Katz J, McGarry JD. The glucosc paradox. Is glucose a substrate for liver metabolism ? J Clin Invest 1984; 74 : 1901-9.

28. Landau BR, Wahren J. Quantification of the pathways followed in hepatic glycogen formation from glucosc. $F A S E B J 1989$; 2 : 2368-75

29. Maycs PA, Laker M. Effects of acutc and long-term fructosc administration on liver lipid metabolism. Prog Biochem Pharmacol $1986 ; 21$ : 33-58.

30. Vrana A, Kazdova L. Effects of dictary sucrose or fructose on carbohydrate and lipid metabolism. Prog Biochem Pharmacol 1986 ; $21: 59-73$

31. Malaissc W, Malaissc-Lagac F, Davies DR, Vandercammen A, Van Schaftingen E. Regulation of glucokinase by a fructosc 1-phosphate-scnsitive protcin in pancrcatic islcts. Eur J Biochem $1990 ; 190$ 539-45.
Visiblement, nous n'en sommes pas encorc au stade où nous pouvons "prédire " l'activité d'une enzyme in vivo à partir de ses propriétés observées in vitro. Bien au contraire, c'est l'approche qui consiste à mesurer dans un premier temps des flux de métabolites dans des cellules intactes, à voir ensuite si l'on rend compte des variations de flux par des changements dans la concentration de certains métabolites effecteurs, à étudier enfin les propriétés cinétiques de l'enzyme dans des extraits ccllulaires rclativement concentrés, qui devrait mener le plus sûrement à la découverte de mécanismes régulateurs analogues à celui décrit pour la glucokinase. Nombre d'enzymes, susceptibles a priori d'ĉtrc assujetties à un contrôle métabolique (parce que catalysant des réactions irréversibles dans des conditions physiologiques), ne semblent pas dotées de propriétés régulatrices. L'avenir nous dira si certaines d'entre elles suivent l'exemple de la glucokinase ou si celle-ci est, au contraire, l'exception

\section{Summary}

A new mechanism for the shortterm regulation of liver glucokinase

Fructose stimulates the phosphorylation of glucose in the liver. The mechanism of this effect involves a regulatory protein, which, in the presence of fructose 6-phosphate, binds to, and inhibits glucokinase. Fructose gives rise to fructose 1-phosphate, which binds to the regulatory protein and causes its dissociation from glucokinase, thus allowing this enzyme to be fully active. Because of this effect, fructose can play the role of a signal which tells the liver that glucose is absorbed from the gut.

\section{TIRÉS A PART}

E. Van Schaftingen. 